\title{
Small cell carcinoma of the lung
}

\author{
P. G. M. MORGAN \\ M.R.C.P. (U.K.)
}

Department of Thoracic Medicine, Brompton Hospital, London

\begin{abstract}
Summary
Over a 5-year period 230 cases of small cell carcinoma presented to Brompton Hospital, London. The male to female ratio was $2.5: 1$ and $39 \%$ had evidence of extrathoracic disease. Forty-five patients were untreated and had a median survival of 70 days. The 5-year survival for surgery and radiotherapy was $6.6 \%$ and $4.2 \%$ respectively. Of 13 patients treated with chemotherapy the median survival was 285 days. The factors which influence survival are described.
\end{abstract}

\section{Introduction}

Survival of small cell carcinoma is very short whether measured from the first symptom or from the date of histological diagnosis (Hyde et al., 1965). Without knowledge of the natural history of the disease evaluation of therapy is impossible - this is especially relevant in view of claims of increased survival by different treatment methods (Broder, Cohen and Selawry, 1977).

Unfortunately, the study of lung cancer is a maze of difficulties. The literature abounds in inconsistencies-criteria for patient selection are rarely specified in detail; description of patients, their disease and therapy is often incomplete and methods of reporting end results vary greatly. Findings are presented with the implied assumption that treated cases survive because of treatment. In order to understand the aetiology of small cell carcinoma and the effects of treatment on it, all the cases presenting to Brompton Hospital, London, over a 5-year period have been reviewed.

\section{Material}

Between January 1970 and January 1975, 230 patients were diagnosed as having small cell carcinoma of the bronchus. Seventy-eight were female and 152 were male. They were followed-up from the first hospital admission until death, or if alive, until January 1978. Eight patients were lost to follow-up.

\section{Results}

Epidemiology

The male to female ratio was $2.5: 1$ and the ages ranged from 12-85 years with a mean of $60.6 \stackrel{\omega}{\circ}$ years: $81 \%$ of the patients were between 50 and 708 years old. The mean age of females and males was 3 . 60.2 years and 60.7 years respectively.

Four were non-smokers ( 2 male and 2 female). Twenty-one per cent. were heavy smokers $(\geqslant 30 \mathrm{\omega}$ cigarettes/day), $50 \%$ were moderate smokers $(15-20 \vec{\circ}$ cigarettes/day) and $29 \%$ were mild smokers $(<15 \mathrm{~N}$ cigarettes/day). Four patients had been exposed to은 asbestos and one had worked in the manufacture of $\rightarrow$ coal dust.

\section{Symptomatology and physical signs}

Ten patients $(4.3 \%)$ were asymptomatic presentation. The mean duration of symptoms of the remaining patients was 1.8 months (range 2 weeks to 9 months), and $20 \%$ complained of one symptom only. The commonest symptoms were cough, $\bar{Q}$ shortness of breath and chest pain. Haemoptysis $\frac{2}{\infty}$

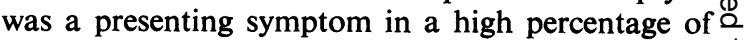
cases $(20 \%)$. Non-specific symptoms were common, $\overrightarrow{\overrightarrow{0}}$ malaise in $20 \%$ and weight loss in $19 \%$. Nine 3 patients were seen following slow resolution of a chest infection and 23 patients $(10 \%)$ complained of symptoms related to extra-thoracic disease.

Clubbing was present in 10 males and 6 females. Thirty-one patients $(13 \%)$ had pleural effusions and $\frac{5}{3}$ 16 patients had superior vena caval obstruction at presentation. Extra-thoracic lymphadenopathy was $\mathrm{O}$ discovered in 50 patients $(22 \%)$ the commonest site being the cervical and supraclavicular nodes $(80 \%)$. 을 Hepatomegaly was found in 41 patients $(18 \%)$. $\rightarrow$ Thirty patients $(13 \%)$ had bone metastases at presentation. Central nervous system involvement $N$ was uncommon-2 patients had cerebral metastases and 2 had a peripheral neuropathy.

\section{Investigations}

Radiologically, 127 patients $(58 \%)$ had right- $\frac{\varrho}{\frac{0}{0}}$ sided disease and two-thirds of these had media- $\stackrel{\infty}{+}$ stinal involvement. Of the remaining 103 patients 0 with left-sided disease, $55(53 \%)$ had mediastinal 0 involvement. 
Histological diagnosis was obtained at bronchoscopy in 162 cases $(70 \%)$, exploratory thoracotomy in 10 cases $(4 \%)$, lymph node biopsy in 5 cases and in the remaining 53 cases $(23 \%)$ by sputum cytology. Skin biopsy provided the diagnosis in one case.

Liver scintiscans were performed in 24 patients and were positive in 18 , correlating with hepatomegaly and abnormal liver function tests. Bone scintiscans were positive in 5 out of 11 performed.

Biochemical studies showed that in 62 out of 214 patients in whom the serum sodium was measured the serum sodium was less than $132 \mathrm{mmol} / \mathrm{l}$ (normal range $132-145 \mathrm{mmol} / \mathrm{l})$. Of these, 12 had an inappropriate ADH secretion (sodium $<125 \mathrm{mmol} / 1$ in the presence of urinary plasma osmolality ratio of $3: 1$ or greater). Eighteen of 213 patients had a serum potassium below the normal limit $(<3.5$ $\mathrm{mmol} / \mathrm{l}$ ) and one patient was found to have an ACTH-like secreting tumour with grossly elevated plasma cortisol. Hypercalcaemia was a feature in 2 patients. Liver function studies (alkaline phosphatase, aspartate transaminase, $\gamma$-glutamyl transpeptidase and bilirubin) were normal in $\mathbf{1 2 5}$ of $\mathbf{1 9 0}$ measurements; 36 patients had elevated alkaline phosphatase and 29 had elevation of all the liver enzymes.

\section{Spread of disease}

Staging was performed according to a modification of the TMN classification (Cohen, 1978):

1. Limited disease confined to one hemithorax without lymph node enlargement.

2. Unilateral lung and mediastinal involvement.

3. Extensive disease (including thoracic wall involvement).

Thirty-two patients $(14 \%)$ had localized disease, $109(47 \%)$ had lung and mediastinal disease and $89(39 \%)$ had extensive disease.

Detailed follow-up was not available in all patients and only 11 post-mortem examinations were performed. Nineteen patients developed brain metastases clinically and 103 had evidence suggestive of liver involvement at death.

\section{Survival}

The survival rate as an actuarial survival, calculated by the life table method (Watson and Berg, 1962) is shown on Table 1. Median survival of the whole group was 5.5 months, one-year survival was $15.8 \%$ and 5 -year survival was $2 \cdot 3 \%$. Shortened survival correlated with increasing age $(P<0.02)$, and low haemoglobin $(P<0.05)$, abnormal liver function studies $(P<0.01)$ and a low serum sodium $(P<0.05)$. Neither the duration of symptoms before diagnosis nor the number of cigarettes smoked altered survival. Signs of extensive disease (lymphadenopathy, hepatomegaly, pleural effusion) did not affect survival.

\section{Treatment}

Untreated

Forty-five patients were untreated (32 male and 13 female), their mean age was 64.5 years (range 45-85 years). Treatment was considered undesirable for 4 reasons: (a) patients who had minimal symptoms or who were asymptomatic at time of diagnosis (20); (b) patients who refused treatment (5); (c) patients with widely disseminated disease and who were too ill (18); (d) patients with other diseases (e.g. obstructive airways disease) (2). Five patients had limited disease, 13 had mediastinal involvement and $27(60 \%)$ had extensive disease.

Survival by actuarial analysis revealed a median survival of 70 days (range $4-485$ days). The one year survival was $6 \%$. The median survival of asymptomatic patients who refused treatment was 112 days whereas if symptomatic it was 28 days. When patients who were asymptomatic were divided into those with localized and extensive disease the median survivals were 122 and 95 days respectively.

\section{Radiotherapy}

Radiotherapy to the primary site and/or mediastinal metastases was received by 124 patients ( 88 male and 36 female; mean age, 59.6 years). Nine patients had limited disease, 75 had intra-thoracic lymph involvement and $36(30 \%)$ had extensive disease. Of this latter group, 19 had extra-thoracic

TABLE 1. Survival of patients with small cell carcinoma

\begin{tabular}{|c|c|c|c|c|c|c|c|c|c|}
\hline \multirow{2}{*}{ Treatment } & \multirow{2}{*}{ Number } & \multirow{2}{*}{$\begin{array}{c}\text { Age } \\
\text { mean }\end{array}$} & \multicolumn{2}{|c|}{ Staging $(\%)$} & \multicolumn{4}{|c|}{$\%$ Survival (months) } & \multirow{2}{*}{$\begin{array}{c}\text { Median } \\
\text { (days) }\end{array}$} \\
\hline & & & $1+2$ & 3 & 4 & 6 & 12 & 60 & \\
\hline Untreated & 45 & $64 \cdot 5$ & 39 & 61 & 31 & 18 & 6 & 0 & 70 \\
\hline Radiotherapy & 124 & $59 \cdot 5$ & 70 & 30 & 70 & 53 & 15 & $4 \cdot 2$ & 174 \\
\hline Surgery & 26 & $57 \cdot 9$ & 100 & 0 & 79 & 67 & 29 & 6.6 & 201 \\
\hline Chemotherapy & 13 & $58 \cdot 7$ & 20 & 80 & 79 & 68 & 28 & 0 & 285 \\
\hline Chemotherapy and radiotherapy & 10 & $58 \cdot 6$ & 23 & 77 & 92 & 69 & 15 & 0 & 248 \\
\hline Palliative radiotherapy & 12 & $59 \cdot 4$ & 0 & 100 & 40 & 10 & 0 & 0 & 74 \\
\hline
\end{tabular}


lymph nodes included in the field of radiotherapy and 4 had hepatomegaly but normal liver function. The median survival was 174 days, at one year $15 \%$ were alive and at 5 years, $4.2 \%$. The dose of radiotherapy varied between 3600 and 4000 rad, usually given in 12 fractions over 28 days.

Palliative radiotherapy for symptomatic relief was given to 13 patients with a survival of 30-179 days. Their survival was significantly shorter than those having curative radiotherapy $(P<0.05)$ and similar to the untreated group.

\section{Surgery}

Of 29 operative procedures performed, 26 cases were operable. Eighteen had limited disease and 8 had mediastinal involvement, none had extensive disease; 14 patients had radical pneumonectomies and 12 had lobectomies and follow-up was available in 24 cases. Their mean age was 59.5 years, significantly younger than the untreated group $(P<0.01)$ but comparable to the radiotherapy group. The median survival was 201 days (range 10.2-190). The one-year survival was $29 \%$ and at 5 years, $6 \cdot 6 \%$.

\section{Chemotherapy}

Thirteen patients were treated with chemotherapy, their mean age was 58.7 years. Two patients had mediastinal metastases and 11 had extensive disease. A varied chemotherapeutic approach was used from single agents to combinations of drugs and therefore in such a small group it was impossible to compare drugs. At one year $20 \%$ were alive but all were dead by 18 months. Compared to the untreated group, survival was prolonged $(P<0.001)$ but, compared to radiotherapy or surgically treated patients, survival was similar.

\section{Radiotherapy and chemotherapy}

Ten patients were treated with combined therapy. At one year, $28 \%$ were alive but all were dead by 15 months. The number was so small that statistical analysis was not performed

\section{Discussion}

Until the 1960s small cell carcinoma was a disease of males with a male to female ratio of $11: 1$ (Bradford Hill, 1966). Over the past 10 years the number of females with the disease has been increasing and the ratio of $2.5: 1$ in this series confirms this trend, perhaps reflecting the alteration in female smoking habits. The fact that $98 \%$ of the patients were smokers provides supporting evidence for the relationship between cigarette smoking and small cell carcinoma (Wynder and Graham, 1950; Doll, Hill and Kreyberg, 1957).

Two notable features of this carcinoma have been confirmed on examination, firstly the rarity of clubbing (Yacoub, 1965) and secondly the widespread nature of the disease at presentation. The $\frac{3}{\mathbb{D}}$ figure of $39 \%$ with extensive disease is similar to the $41 \%$ found in a recent study of 56 patients from Brompton hospital, when staging included scinti- $\overrightarrow{\overrightarrow{\vec{S}}}$ scans (bone, liver) and bone marrow aspiration $\overrightarrow{0}$ (Gregor et al., 1979). American series report that $\frac{\bar{\sigma}}{\mathrm{a}}$ $60-70 \%$ of patients have dissemination at diagnosis and suggest that either the disease is different in the $\mathbb{\varnothing}$ U.S.A. or that more likely patients present at a later time in the course of their disease (Hansen, Muggia क and Selawry, 1971).

The course of a disease in a patient not treated by $\overrightarrow{\vec{\omega}}$ potentially curative procedures illustrates the natural $\stackrel{\omega}{\mathcal{D}}$ history of the disease in that patient, the course in a $\bar{Q}$ group of unselected untreated patients would there- 3 fore be expected to provide an expression of it. It is ir impossible to find such a group of patients in the literature, patients being untreated because they were of too ill or had widely disseminated disease. The discovery of such a high percentage of untreated $\stackrel{N}{N}$ patients in this series allows study of the disease 음 without recourse to historical reports where small $\rightarrow$ cell carcinoma was classified under the heading of $\vec{z}$ anaplastic cell carcinoma. Unfortunately, the untreated group were selective, being older and with $\stackrel{\rho}{\supset}$ more disseminated disease than the treated group $\overrightarrow{0}$ However, it is interesting to find that if asympte matic, the median survival was 4 months, rath longer than the usually quoted figures of 2 months and poses the question that if more asymptomatic patients had been untreated would the median survival have approached the figures quoted for $\frac{\varnothing}{\varnothing}$ treatment groups?

It is therefore important to discover what features influence survival. Bignall (1955) suggested 3 factors: (a) the age of the patient-the older the patient the longer the survival; (b) position of tumour in the lung, underlined in a recent review (Peters, 1977); 윽 (c) the malignant capabilities of the tumour. In this series age was related to survival, i.e. older patients had a reduced survival. Position of the tumour did not affect survival, confirming the finding in the $\frac{\rho}{3}$ MRC trial in treatment of small cell carcinoma (Miller, Fox and Tall, 1969). The malignant capability of a tumour is difficult to define, on one hand $\frac{D}{O}$ it may represent tumour dissemination and indirectly tumour growth; on the other, the ability to release of substances from the tumour either as ectopic $N$ hormones or tumour markers. Only one parameter 0 of dissemination correlated with a reduction in $\underset{\omega}{\sigma}$ survival-liver involvement. Why this should be the case is not known but this has been described as a determinant of response to treatment in a previous study (Gregor and Morgan, 1979). Equating dis- $\stackrel{+}{?}$ semination with reduced survival is dangerous as it ${ }_{0}^{0}$ excludes consideration of other factors which may $\vec{\Phi}$ 
explain why patients with purely intrathoracic disease have similar survivals as those with widespread disease. These include ectopic hormone production, patients with ACTH-like and inappropriate ADH syndromes have a shortened survival $(P<0.01)$, and tumours with rapid doubling times. So far, tumour markers have not been helpful in the study of the natural history of this disease.

The fundamental question to be answered is 'Does treatment increase survival?' There are few controlled trials which compare treated with untreated patients. Radiotherapy achieves an objective regression in tumour size in $80 \%$ of cases (King et $a l ., 1977)$, and since the role of surgery has been questioned since the MRC trial, it has remained until recently the treatment of choice. The survival figures in this study (Table 1) reveal no significant difference between these treatment modalities, with median survivals of 6 months and 7 months for surgery and radiotherapy respectively. The survival figures are statistically significant when compared to untreated patients $(P<0.001)$ but in real terms they represent an increased survival of 2 months when compared to the asymptomatic untreated patients. Although the 5-year survival of the surgical group of $6.6 \%$ differs from the figure $(0 \%)$ obtained in the MRC trial (Miller et al., 1969) the numbers involved represent only $12.6 \%$ of the total patients treated, reflecting the small number of patients who have localized disease and who are fit for surgery. In comparison to patients treated with radiotherapy $(53 \%), 30 \%$ had extra-thoracic disease and the 5 -year survival was similar at $4 \cdot 2 \%$.

Chemotherapy has been introduced as a means of treating disseminated disease and many trials have shown tumour responsiveness (Hansen et al., 1976; Selawry and Hansen, 1973). In the very small group of patients treated in the present trial, the median survival of patients was 285 days although $80 \%$ had extra-thoracic disease. This compares well with radiotherapy and surgery, although there were no long-term survivors.

Before treatment can be presented as increasing survival the aetiology of small cell carcinoma must be recognized. The wide variation in the disease process in untreated and treated patients suggests that this is a heterogeneous disease and that tumours behave kinetically, biologically and morphologically in different ways. Until these factors are recognized the ideal treatment will remain unknown. The place of surgery in localized disease, radiotherapy in palliation and extensive local disease, and chemotherapy in systemic disease must remain subjects for future trials.

\section{References}

Bignall, J.R. (1955) Natural duration of bronchial carcinoma. Lancet, ii, 210.

Bradford Hill, A. (1966) Principles of Medical Statistics. p. 220. London.

Broder, E., Cohen, M.H. \& Selawry, O.S. (1977) Treatment of bronchogenic carcinoma; II, Small cell. Cancer Treatment Reviews, 4, 219.

Doll, R., Hill, A.B. \& KREYBerg, L. (1957) The significance of cell type in relation to the aetiology of lung cancer. British Journal of Cancer, 11, 43.

Gregor, A., Morgan, P.G.M., Morgan, R.L., Scadding, F.H.\& TURNER-WARWICK, M. (1979) Small cell carcinoma: combined approach to treatment. Thorax, 34, 789.

Gregor, A. \& MORGAN, P.G.M. (1979) Factors influencing response to combination chemotherapy in small cell carcinoma of the bronchus. Proceedings of $\mathrm{ASCO}, \mathrm{New}$ Orleans, (May).

Hansen, H.H., Muggia, F.M. \& Selawry, O.S. (1971) Bone marrow examination in 100 consecutive patients with bronchogenic carcinoma. Lancet, 2, 443.

Hansen, H., Selawry, O.S., Simon, R., Carr, D.T., Van WYK, C.E., TUCKER, R.D. \& SEALY, R. (1976) Combination chemotherapy of advanced lung cancer. Cancer, 38, 220.

Hyde, L., Yee, J. \& Wilson, Patno, M.E. (1965) Cell type and the natural history of lung cancer. Journal of the American Medical Association, 193, 52.

King, G.A., Comis, R., Ginsberg, S., Goldberg, J., Dale, H.T., Brown, J., Dalel, P., Chung, C. \& Gottlieb, A. (1977) Combination chemotherapy and radiotherapy in small cell carcinoma of the lung. Radiology, 125, 529.

Miller, A.B., FoX, W. \& TALL, R. (1969) Five year follow-up of the Medical Research Council comparative trial of

$\because$ surgery and radiotherapy for the primary treatment of small celled or oat celled carcinoma of the bronchus. Lancet, ii, 501.

Peters, R.M. (1977) Staging of lung cancer. Chest, 71, 633.

SELAWRY, O.S. \& HANSEN, H.H. (1973) In: Cancer Medicine, (Ed. by Holland, J.F. \& Frei, E. (III)), p. 1473. Lea and Febiger, Philadelphia.

WATSON, W.L. \& Berg, J.W. (1962) Oat cell cancer. Cancer, $15,759$.

WyNDER, E.L. \& GRAHAM, E.A. (1950) Tobacco smoking as a possible etiologic factor in bronchogenic carcinoma-a study of 684 proved cases. Journal of the American Medical Association, 143, 329.

YACOUB, M.H. (1965) Relations between the histology of bronchial carcinoma and hypertrophic pulmonary osteqarthropathy. Thorax, 20, 537, 